\title{
Application of an Improved Ensemble Local Mean Decomposition Method for Gearbox Composite Fault Diagnosis
}

\author{
Zhijian Wang $\mathbb{D}^{1,2}$, Junyuan Wang $\left(\mathbb{D},{ }^{1}\right.$ Wenan Cai $\left(\mathbb{D},{ }^{3}\right.$ Jie Zhou $\mathbb{D}^{1},{ }^{1}$ Wenhua Du, ${ }^{1}$ \\ Jingtai Wang $\left(\mathbb{1},{ }^{1}\right.$ Gaofeng $\mathrm{He}\left(\mathbb{B},{ }^{1}\right.$ and Huihui He $\mathbb{1}^{1}$ \\ ${ }^{1}$ School of Mechanical Engineering, North University of China, Taiyuan, Shanxi 030051, China \\ ${ }^{2}$ School of Mechanical Engineering, Xian Jiaotong University, Shanxi 030619, China \\ ${ }^{3}$ School of Mechanical Engineering, Jinzhong University, Jinzhong, Shanxi 030600, China \\ Correspondence should be addressed to Junyuan Wang; wangyi01161013@163.com and Wenan Cai; caiwenan0008@link.tyut.edu.cn
}

Received 21 January 2019; Revised 9 March 2019; Accepted 2 May 2019; Published 28 May 2019

Academic Editor: Sing Kiong Nguang

Copyright (C) 2019 Zhijian Wang et al. This is an open access article distributed under the Creative Commons Attribution License, which permits unrestricted use, distribution, and reproduction in any medium, provided the original work is properly cited.

\begin{abstract}
In industrial production, it is highly essential to extract faults in gearbox accurately. Specifically, in a strong noise environment, it is difficult to extract the fault features accurately. LMD (local mean decomposition) is widely used as an adaptive decomposition method in fault diagnosis. In order to improve the mode mixing of LMD, ELMD (ensemble Local Mean Decomposition) is proposed as local mode mixing exists in noisy environment, but white noise added in ELMD cannot be completely neutralized leading to the influence of increased white noise on PF (product function) component. This further leads to the increase in reconstruction errors. Therefore, this paper proposes a composite fault diagnosis method for gearboxes based on an improved ensemble local mean decomposition. The idea is to add white noise in pairs to optimize ELMD, defined as CELMD (Complementary Ensemble Local Mean Decomposition) then remove the decomposed high noise component by PE (Permutation Entropy) while applying the SG (Savitzky-Golay) filter to smooth out the low noise in PFs. The method is applied to both simulated signal and experimental signal, which overcomes mode mixing phenomenon and reduces reconstruction error. At the same time, this method avoids the occurrence of pseudocomponents and reduces the amount of calculation. Compared with LMD, ELMD, CELMD, and CELMDAN, it shows that improved ensemble local mean decomposition method is an effective method for extracting composite fault features.
\end{abstract}

\section{Introduction}

When a gearbox fails, the detection signal usually exhibits nonlinear and nonstationary characteristics $[1,2]$. Combined Time-Frequency Analysis is a hotspot of signal processing research $[3,4]$. It can provide information both in the time domain and the frequency domain, which is a vital method of fault diagnosis [5, 6]. Previously employed methods include window Fourier transform [7], Continuous Wavelet Transform [8, 9], Wigner-Ville distribution [10], and Stransformation [11]. However, these methods have several limitations. The width of window function in window Fourier transform is unchangeable [12]. The function of localization in time and frequency domain is not executed simultaneously $[13,14]$. Even though Continuous Wavelet Transform (CWT) is capable of observing the time and frequency information of signals at the same time, it is not suitable as an adaptive processing method.

Empirical Mode Decomposition (EMD) is an adaptive time-frequency processing method, which is often used in the analysis and processing of nonlinear and nonstationary signals $[15,16]$. This method can promptly decompose complex signals into a finite number of Intrinsic Mode Functions (IMF). The IMF components consist of the local characteristic signals of the original signal at different time scales. The complete time-frequency spectrum of the original signal is obtained by Hilbert transform of each eigenmode function $[17,18]$. Although the adaptive EMD method is able to obtain the complete time frequency distribution of the original signal, there are certain limitations such as undershoots, overshoots, edge effects, and mode mixing [19]. Mode mixing refers to the fact that one component of the 
IMF may contain different time scales, or the same time scale may decompose into different mode components. Noise interference, intermittent signals, and pulse interference all cause mode mixing. Colominas and Wang [20,21] proposed to add white noise to the original signal to improve the accuracy of EMD decomposition; Wu et al. [22] proposed Ensemble Empirical Mode Decomposition (EEMD), which is performed after different white noises are added to the original signal. The corresponding components obtained by multiple decompositions are averaged to obtain the final IMF component. However, the added white noise amplitude and the number of integrations affect the decomposition accuracy of the EEMD algorithm $[23,24]$. There is no available formula that can determine this parameter; therefore, Yeh et al. [25] proposed Complementary Ensemble Empirical Mode Decomposition (CEEMD), which first adds two opposite white noise signals to the original signal and then decompose by EMD, and CEEMD reduces the reconstruction error caused by white noise. However, CEEMD not only increases the amount of computation, but also produces more pseudocomponents when the added magnitude and number of iterations are not appropriate.

Local Mean Decomposition (LMD) is an adaptive processing method for nonlinear and nonstationary signals proposed by Jonathan S. Smith [26]. LMD can decompose nonstationary and multicomponent signals into several Product Functions (PFs). The instantaneous frequency of each PF has a physical significance, and each PF component corresponds to a certain physical process. The PF is a single-component amplitude modulation-frequency modulation (AM-FM) signal, so the essence of LMD is to adaptively decompose a multicomponent signal into multiple single-component AMFM signals, which makes LMD especially suitable for dealing with nonstationary and nonlinear signals. Comparing LMD to EMD $[27,28]$ shows that LMD can suppress the endpoint effect to a certain extent and has the advantages of less false components and fewer iterations. However, in the fault feature extraction, noise may be distributed to make the decomposition result exhibit mode mixing.

In recent years, LMD has been widely used in damage identification. Wang et al. [29] have combined local mean decomposition and energy dispersion rate for low-speed helical gearbox fault diagnosis. Wang [30] has proposed a bearing fault diagnosis method based on local mean decomposition and multiscale entropy. Liu [31] has combined local mean decomposition and kernel principal component analysis and applied to fiber optic gyroscope vibration error analysis. To address the problem of mode mixing in LMD, Chen et al. [32] have proposed an overall local mean decomposition method based on noise-assisted analysis, namely ensemble local mean decomposition (ELMD). It adds white noise with finite amplitude to the original signal, then decomposes the signal with white noise by LMD, repeats the above process many times, adds different white noise to the original signal each time, and finally calculates the average of all the decomposed PF components to get the final decomposition result. However, ELMD is limited by the number of white noise additions, so the completeness of ELMD is poor because the white noise cannot be completely neutralized, and false components will appear when the selection of the amplitude and iteration number of white noise additions is inappropriate. Moreover, the added white noise being random the signals decomposed by ELMD are different every time. Therefore, after ELMD decomposition of these different signals, different decomposition layers will be obtained. In order to solve the above problems of ELMD, the first method is to fill the missing PF component with a time series of amplitude 0. However, this may cause the last few PFs to have almost no energy and hardly represent the relevant information of the signal. The second method is to set a certain number of layers so that ELMD decomposes the same number of layers each time, but this will cause ELMD to be no longer fully adaptive.

Based on the above analysis, this paper proposes a gearbox composite fault feature extraction method based on an improved ELMD. Considering that permutation entropy (PE) can effectively amplify the weak changes in a time series, it has important application in signal mutation detection. The entropy can reflect the uncertainty of the time series: the smaller the randomness of the time series, the smaller is the obtained entropy, and the larger the randomness of the time series, the larger is the obtained entropy $[33,34]$. Hence the abnormal signal can be removed by calculating the permutation entropy. In addition, the SG filter is used to remove the noise in the low noise component. The SG filter is a best fit in the time domain by using the least square method through the moving window. It can effectively remove the noise without changing the shape and width of the original signal [35]. First, the original signal is decomposed by CELMD, and then the abnormal signals are detected using permutation entropy. The normal signal is decomposed by LMD after being reconstructed. Since most of the noise is filtered by CELMD and permutation entropy, the PF component obtained will contain only a small amount of noise, the residual noise is removed by SG filtering to obtain the final decomposition result.

In summary, considering that CELMD can eliminate abnormal signals makes LMD overcome the mode mixing phenomenon and reduce the reconstruction error. Permutation entropy can measure the noise level of the signal, which can avoid the occurrence of pseudocomponents and reduce the calculation amount. Finally, the residual noise is removed by SG filtering. In this paper, CELMD, permutation entropy, and SG filtering are combined to obtain the gearbox fault feature extraction method based on improved ELMD. The effectiveness of the proposed method is proved by its application on the simulated signal and the measured signal.

\section{Theory Behind the Methods}

2.1. Permutation Entropy. Permutation Entropy (PE) is an average entropy function used to measure the complexity of a one-dimensional time series. This function is highly sensitive to signal transformation and can amplify the microsignal in the system. This method can detect the dynamic mutation of complex system well and can also detect the nonlinear and nonstationary signals. Permutation entropy has been widely 
used in medicine, meteorology, and other fields, and now it is gradually being applied to mechanical fault diagnosis. The basic algorithm for permutation entropy is [36]

(1) Phase Space Reconstruction of Time Series. A phase sequence can be obtained by performing phase space reconstruction on a time series $\{x(i), i=1,2, \ldots, n\}$ with a length of $\mathrm{N}$ :

$$
\begin{aligned}
Y & =\left[\begin{array}{c}
Y(1) \\
Y(2) \\
\vdots \\
Y(j) \\
\vdots \\
Y(K)
\end{array}\right] \\
& =\left[\begin{array}{cccc}
x(1) & x(1+\tau) & \cdots & x(1+(m-1) \tau) \\
x(2) & x(2+\tau) & \cdots & x(2+(m-1) \tau) \\
\vdots & \vdots & & \vdots \\
x(j) & x(j+\tau) & \cdots & x(j+(m-1) \tau) \\
\vdots & \vdots & & \vdots \\
x(K) & x(K+\tau) & \cdots & x(K+(m-1) \tau)
\end{array}\right]
\end{aligned}
$$

Here $j=1,2,3, \ldots, K ; K+(m-1) \tau=n ; m$ is the embedded dimension and $\tau$ is the delay time. In the reconstruction matrix $\mathrm{Y}$, each $\mathrm{Y}(\mathrm{j})$ is a reconstructed component, so there are $\mathrm{K}$ reconstruction components in $\mathrm{Y}$.

(2) Arrange the Reconstructed Components in Ascending Order. Rearrange the $j$ th reconstructed component in $Y(j)=$ $\{x(j) x(j+\tau) \cdots x(j+(m-1) \tau)\}$ in ascending order, and the position of each element in the reconstructed component is referenced by $i_{1}, i_{2}, \ldots, i_{m}$. That is

$$
\begin{aligned}
x\left(j+\left(i_{1}-1\right) \tau\right) & \leq x\left(j+\left(i_{2}-1\right) \tau\right) \leq \cdots \\
& \leq x\left(j+\left(i_{m}-1\right) \tau\right)
\end{aligned}
$$

If there are equal values in the reconstructed component, which is

$$
x\left(j-\left(i_{p}-1\right) \tau\right)=x\left(j-\left(i_{q}-1\right) \tau\right)
$$

then, follow $i_{p}$ with $i_{q}$, that is to say, if $i_{p}<i_{q}$, then

$$
x\left(j-\left(i_{p}-1\right) \tau\right) \leq x\left(j-\left(i_{q}-1\right) \tau\right) .
$$

Therefore, for any reconstructed component $Y(j)$ of the reconstruction matrix $\mathrm{Y}$, a set of sequences can be obtained: $S(j)=\left(i_{1}, i_{2}, \ldots, i_{m}\right) j=1,2, \ldots, k$. It is thought that there are $\mathrm{m}$ ! kinds of index sequences of different positions mapped by $\mathrm{m}$-dimensional vector space, and the same ascending order sequence may exist in each reconstructed component, so $\mathrm{k} \leq$ $\mathrm{m} !$.
(3) Calculate Value of Permutation Entropy. Let the probability of each position index sequence appear as $p_{1}, p_{2}, \ldots, p_{k}$. According to the form of entropy, permutation entropy (PE) of the $\mathrm{k}$ different index sequences of the time series is defined as

$$
H_{p}(m)=-\sum_{i=1}^{k} p_{i} \ln p_{i}
$$

(4) Normalization of Permutation Entropy. The normalization of $H_{p}$ can make the comparison of permutation entropy more convenient. $H_{p}$ is normalized using $\mathrm{m}$ !:

$$
H_{p}=\frac{H_{p}(m)}{\ln m !}
$$

where $0 \leq H_{p} \leq 1$.

The value of $H_{p}$ reflects the degree of randomness of the time series $\{\mathrm{x}(i), i=1,2, \ldots, n\}$. If the time series is more regular, $H_{p}$ is smaller and vice versa. The changes in $H_{p}$ magnify small changes in the time series.

2.2. SG Filtering. The Savitzky-Golay filter is widely used for data stream smoothing and noise reduction. The specific algorithm is as follows [37].

Each $\mathrm{M}$ sample points near $\mathrm{x}$ in the original data are taken, and $\mathrm{x}$ is set as the origin. That is, an array of windows containing $2 \mathrm{M}+1$ sample points centered on $\mathrm{x}$ is constructed and an i-order polynomial is constructed to fit the array:

$$
p(n)=\sum_{k=0}^{i} a_{k} \cdot n^{k}
$$

where $-M \leq n \leq M, i \leq 2 M+1$

The fitted residual is

$$
\varepsilon=\sum_{n=-M}^{M}(p(n)-x(n))^{2}=\sum_{n=-M}^{M}\left(\sum_{k=0}^{i} a_{k} \cdot n^{k}-x(n)\right)^{2}
$$

The smaller the $\varepsilon$, the higher the fit to the original data. In order to minimize $\varepsilon$, the partial derivative of $\varepsilon$ for each parameter is 0 :

$$
\frac{\partial \varepsilon}{\partial a_{i}}=\sum_{n=-M}^{M} 2 n^{i}\left(\sum_{k=0}^{N} a^{k} \cdot n^{k}-x[n]\right)^{2}
$$

That is,

$$
\sum_{k=0}^{N}\left(\sum_{n=-M}^{M} n^{i+k}\right) a_{k}=\sum_{n=-M}^{M} n^{i} x[n]
$$

Then this window array is moved until all the fit points of the original data are obtained. In this process, the noise portion deviating from the normal curve trend is removed, so the method has a smooth filtering effect on the data. In this paper, SG filtering is used to remove noise in low noise components. As shown in Figure 1, (a) is the original signal x, (b) is the signal $\mathrm{xl}$ after the high noise component is removed 


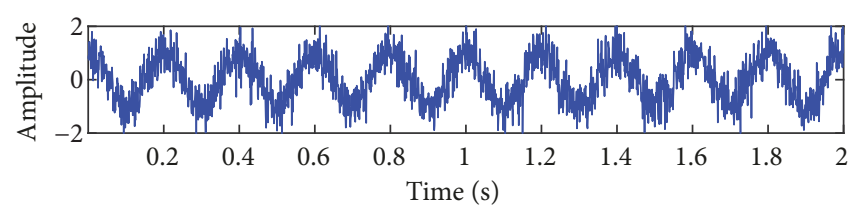

(a) Original signal

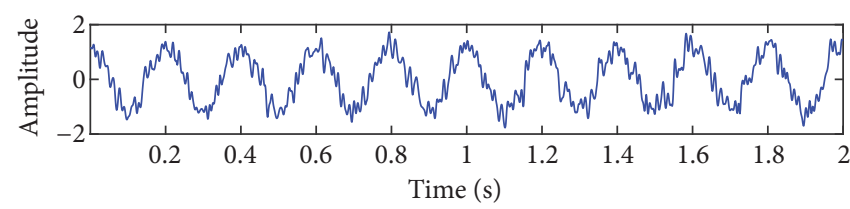

(b) Signal after removing high-frequency noise by auxiliary noise and PE

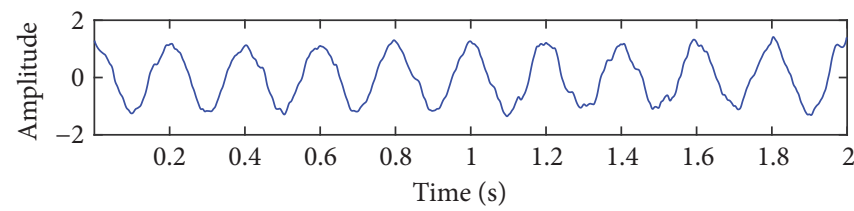

(c) Results after further filtering through SG

FIGURE 1: Noise-containing signal and noise-reduced signal.

by the noise and the Permutation entropy, and (c) is the result of smoothing $\mathrm{xl}$ by the SG filter. It can be seen that most of the noise has been removed by the auxiliary noise and permutation entropy, and the noise in the original signal can be almost completely eliminated by S-G filtering.

\subsection{Improved Ensemble Local Mean Decomposition Method.} There are three parameters of permutation entropy in CELMD that need to be determined: length of the time series $(\mathrm{N})$, embedding dimension $(m)$, and time delay $(\lambda)$.

Time series length $\mathrm{N}$ : the time series length used here is $\mathrm{N}=2000$.

Embedding dimension $m$ : in the calculation of PE, if the value of $m$ is too large, the time series will be homogenized after the reconstruction of phase space, which will increase the amount of calculation and cannot reflect the subtle changes of the sequence. On the contrary, if $\mathrm{m}$ is too small, it will cause the reconstructed vector to contain few states, making the algorithm meaningless. Bandt suggests that the value of the embedded dimension $m$ be taken as $3 \sim 7[38,39]$. When the data length is small, the value of the embedded dimension is smaller. When the data length is larger than 720 , $m=5$ works well.

Time delay: the impact of the choice of time delay on the calculation is very small; this paper takes $\lambda=1$.

After calculating the permutation entropy of a component, it is necessary to determine whether it is a high noise component. In this case, a threshold $\theta_{0}$ needs to be set. This paper selects $\theta_{0}$ by calculating the permutation entropy of different signals.

The permutation entropy values of representative signals are calculated as follows:

$$
\begin{aligned}
& x_{1}(t)=(1+\sin (5 \pi t)) \sin (50 \pi t) \\
& x_{2}(t)=\sin (50 \pi t) \\
& x_{3}(t)=\sin (100 \pi t) \sin (100 \pi t) \\
& x_{4}(t)=\sin (300 \pi t)
\end{aligned}
$$

TABLE 1: PE values corresponding to different signals.

\begin{tabular}{lccccccc}
\hline Signal & $x_{1}$ & $x_{2}$ & $x_{3}$ & $x_{4}$ & $x_{5}$ & $x_{6}$ & $x_{7}$ \\
\hline PE & 0.2923 & 0.3031 & 0.3826 & 0.4946 & 0.7043 & 0.9684 & 0.9735 \\
\hline
\end{tabular}

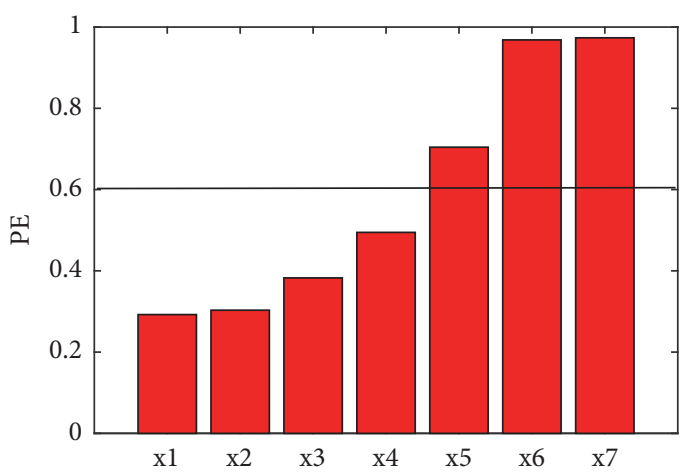

FIGURE 2: PE values corresponding to different signals.

$$
x_{5}(t)=\left[\begin{array}{c}
z \operatorname{eros}(1,300) \\
\operatorname{randn}(1,600) \\
z \operatorname{eros}(1,300) \\
\operatorname{randn}(1,600) \\
z \operatorname{zeros}(1,200)
\end{array}\right]^{T}
$$

$x_{6}(t)$ is white noise, and $x_{7}(t)$ is Gaussian white noise.

The computed permutation entropy values of the above seven signals are shown in Table 1.

The PE corresponding to each simulation signal is shown in Figure 2. It can be seen from the above calculation results that PE of sinusoidal signal and the amplitude modulation signal is small; all are less than 0.6. The intermittent signal is relatively random with respect to the sinusoidal signal, and $\mathrm{PE}$ is 0.7043 . The PE value of white noise and Gauss white noise is larger, and the PE of intermittent signal and noise are all greater than 0.6 . 
TABLE 2: The permutation entropy values corresponding to different noise amplitude.

\begin{tabular}{lccccc}
\hline Amplitude & 0.3 & 0.5 & 0.8 & 1 & 1.5 \\
\hline $\mathrm{PE}$ & 0.7832 & 0.8024 & 0.8616 & 0.9027 & 0.9732 \\
\hline
\end{tabular}

TABLE 3: The permutation entropy values corresponding to different frequency of modulation signal.

\begin{tabular}{lccccc}
\hline Amplitude & 80 & 120 & 180 & 240 & 300 \\
\hline $\mathrm{PE}$ & 0.4012 & 0.4065 & 0.4103 & 0.4151 & 0.4198 \\
\hline
\end{tabular}

TABLE 4: The permutation entropy values corresponding to different amplitude of impulse signal.

\begin{tabular}{lccccc}
\hline Amplitude & 1.5 & 2 & 2.5 & 3 & 3.5 \\
\hline $\mathrm{PE}$ & 0.4835 & 0.4856 & 0.4894 & 0.4954 & 0.5011 \\
\hline
\end{tabular}

In order to make the experiment reliable, $\mathrm{PE}$ of signals with different known energies are solved. Here, we selected the classic model of gearbox fault for simulation verification including modulation signals, impulse signals, and noise [40]. As the noise amplitude increases, PE also increases gradually, remaining greater than 0.6. When the amplitude and frequency of the modulated signal and the impulse signal change, the change of PE is small, and both are less than 0.6. As shown in Tables 2-4.

It can be seen that randomness detection based on permutation entropy can be used to detect abnormal signals and is more appropriate to take $0.55 \sim 0.6$. In this paper, threshold $\theta_{0}=0.6$. If the $\mathrm{PE}$ value of the component is greater than 0.6 , it is considered to be a high noise component.

CELMD adds both positive and negative white noise to the original signal. By adding white noise in pairs, the influence of noise on the original signal can be eliminated and the noise can be reduced.

In the two methods of CELMD and ELMD, the purpose of adding white noise is to change the distribution of signal extreme points and cover abnormal signals such as highfrequency intermittent and noise in the original signal and result in abnormal signals because of mode mixing. The added white noise is decomposed preferentially. After the abnormal signal is removed, the extreme points will be evenly distributed, so it is no longer necessary to add white noise for integration and average decomposition. The specific steps of improved ELMD are as follows:

(1) In the original signal $x(t)$, add white noise signal $n_{i}(t)$, $-n_{i}(t)$ with a mean of 0 ,

$$
\begin{aligned}
& f_{i}^{+}(t)=x(t)+h_{i} \cdot n_{i}(t) \\
& f_{j}^{-}(t)=x(t)-h_{j} \cdot n_{j}(t)
\end{aligned}
$$

where $h_{i}$ and $h_{j}$ control the white noise amplitude; $i$ and $j=$ $1, \ldots, M, M$ is the number of white noise pairs added.

(2) As for $f_{i}^{+}(t)$ with $f_{i}^{-}(t)$, perform LMD decomposition separately to obtain the first layer $\mathrm{PF}$ component $P_{i, 1}^{+}(t)$ and $P_{i, 1}^{-}(t)$.
(3) Integrate the average of the above components:

$$
P_{1}(t)=\frac{1}{2 M} \sum_{i=1}^{M}\left[P_{i, 1}^{+}(t)+P_{i, 1}^{-}(t)\right]
$$

(4) Calculated entropy value of $P_{1}(t)$ and determine whether the component is a high noise component. If the entropy value $\theta_{1}>\theta_{0}$, it is considered to be a high noise component.

(5) If $P_{1}(t)$ is a high noise component, calculated $P_{2}(t), P_{3}(t), \ldots$ according to steps (2)-(4) until it appears $\theta_{m} \leq$ $\theta_{0}$. This is easy to understand that $P_{m}(t)$ is not a high noise component.

(6) Separate high noise $P_{1}(t), \ldots, P_{m-1}(t)$ from the original signal, the signal with low noise forms a new reconstructed signal:

$$
y(t)=x(t)-\sum_{i=1}^{m-1} P_{i}(t)
$$

Decompose the reconstructed signal $y(t)$ with LMD to obtain the PF component.

(7) After the high noise component is removed by CELMD and permutation entropy, there will still be a small amount of noise in the signal and a small amount of error due to the noise, so the low noise component is smoothed by SG filtering. The final decomposition result is obtained. The flow chart of the improved ELMD method is shown in Figure 3.

\section{Simulation Analysis}

Given a simulated signal, as shown in (15), it includes an intermittent signal, a modulated signal, and a periodic shock signal.

$$
\begin{aligned}
x_{1}(t)= & \cos (400 \times 2 \pi t) \\
& \times\left(\begin{array}{c}
u(t)-u(t-0.1)+ \\
u(t-0.2)-u(t-0.3)+ \\
u(t-0.4)-u(t-0.5)
\end{array}\right) \\
x_{2}(t)= & \sin (10 \times 2 \pi t) \times \sin (100 \times 2 \pi t) \\
x_{3}(t)= & 4 \times \exp (0.015 \times 1500 \times 2 \pi t) \\
& \times \sin \left(\sqrt{1-0.015^{2}} \times 1500 \times 2 \pi t\right) \\
x(t)= & x_{1}(t)+x_{2}(t)+x_{3}(t)
\end{aligned}
$$

The time-domain waveform of simulation signal $x$ is shown in Figure 4.

The results obtained by CELMDAN are shown in Figure 5. It can be seen that the decomposition result of this method is almost complete, so the method can determine the $\mathrm{PF}$ of each layer in a noiseless environment. However, in a noisy environment, this method cannot get ideal decomposition results such as adding noise of amplitude 1 to the signal, to obtain the composite signal shown in Figure 6. 


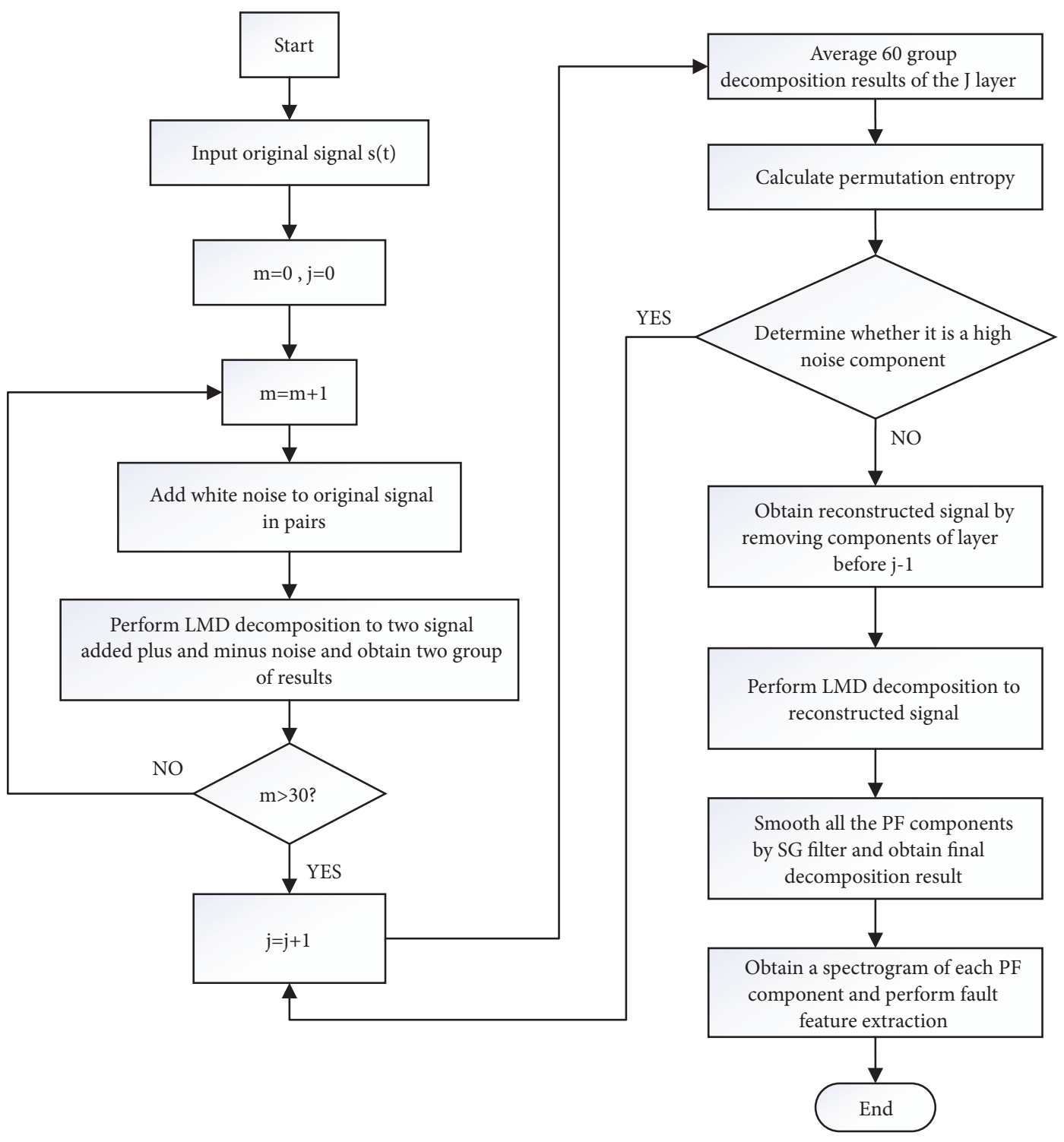

FIGURE 3: Improved ELMD method flow chart.

The signal is decomposed by this method, and the result is shown in Figure 8. As shown in Figure 7, the decomposition result is greatly different from the three components of the original signal and that mode mixing is present, further indicating that CELMDAN does not denoise the original signal when adding white noise. Improved ELMD can reduce the influence of noise on the decomposition result by using permutation entropy. The signal is decomposed by this method, and the result is shown in Figure 8. Compared with CELMDAN, which adds white noise to each PF component, PECELMD no longer adds noise to the signal after removing the high noise component, but uses SG filter to remove the noise in the low noise component, so the reconstruction error is smaller. Therefore, the fault feature can be extracted in a strong noise environment. Compared to CELMDAN, this method has higher decomposition accuracy.
In order to verify the effectiveness of the improved ELMD, nonlinear simulation signal with mixed random noise is used, which is a mixture of three signals, and the three signals are as shown in (16).

$$
\begin{aligned}
& x_{1}=\cos (100 \cdot \pi \cdot t) \\
& x_{2}=(1+t) \cdot \cos (20 \cdot \pi \cdot t) \\
& x_{3}=0.4 \cdot \operatorname{randn}(\operatorname{size}(t))
\end{aligned}
$$

The time-domain wave forms of the three signals are shown in Figure 9, the time-domain waveform of the composite signal is shown in Figure 10.

Decomposition process is as follows by improved ELMD.

(1) Adding 30 pairs of white noise signals with a mean value of 0 to the combined signal $f_{i}^{+}(t)$ with $f_{i}^{-}(t)$, among them $i=1,2, \ldots, 30$. 


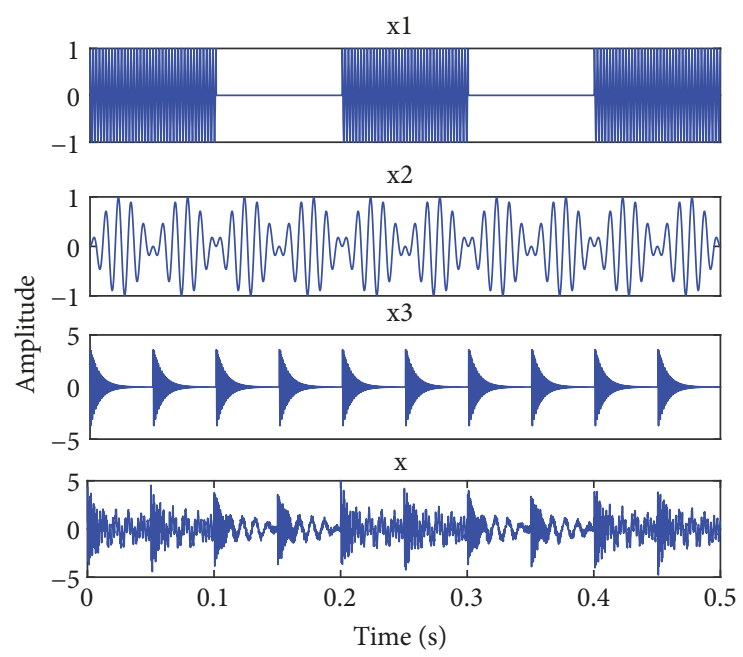

FIGURE 4: Simulation signal time-domain waveform.

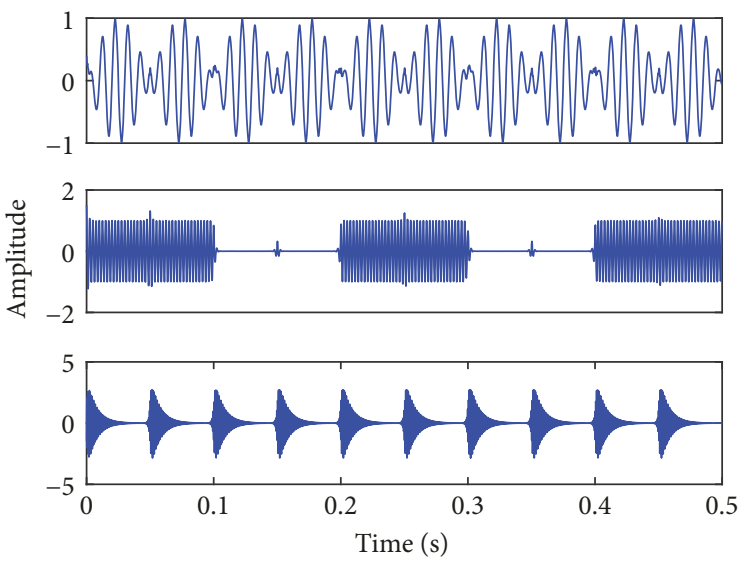

FIgURE 5: CEELMDAN decomposition results.

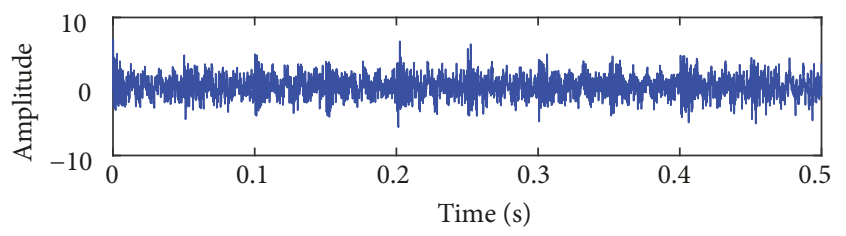

FIGURE 6: Simulation signal after adding noise.

(2) Decompose the signal $\mathrm{x}$ by LMD and PF components $P_{i, \mathrm{j}}^{+}(t)$ with $P_{i, \mathrm{j}}^{-}(t)$ are obtained, where $j$ represents the $j$ th layer $\mathrm{PF}$ component. Average the PF component of the first layer $P_{1}(t)$. The result is shown in Figure 11(a), and the permutation entropy of the first layer PF component is calculated $\theta_{1}=$ $0.9029, \theta_{1}>\theta_{0}$, the first layer PF is the abnormal component.

$$
P_{1}(t)=\frac{1}{60} \sum_{i=1}^{30}\left[P_{i, 1}^{+}(t)+P_{i, 1}^{-}(t)\right]
$$

(3) Continue to average the PF component of the second layer $p_{2}(t)$. The result is shown in Figure 11(b), calculating the entropy $\theta_{2}$ which is 0.7196 . Because $\theta_{2}>\theta_{0}$, so the second layer is also an abnormal signal.

$$
P_{2}(t)=\frac{1}{60} \sum_{i=1}^{30}\left[P_{i, 2}^{+}(t)+P_{i, 2}^{-}(t)\right]
$$

(4) Continue to average the PF component of the third layer $p_{3}(t)$, and the result is shown in Figure 11(c), and the permutation entropy is calculated. $\theta_{3}$ is 0.3438 because $\theta_{3}>$ $\theta_{0}$, so the third layer is the normal signal.

$$
P_{3}(t)=\frac{1}{60} \sum_{i=1}^{30}\left[P_{i, 3}^{+}(t)+P_{i, 3}^{-}(t)\right]
$$

(5) Obtain $\mathrm{PF}_{1}$ and $\mathrm{PF}_{2}$ as an abnormal signal and remove it from the original signal.

The time-domain waveform of the reconstructed residual signal is shown in Figure 12.

Performing LMD decomposition on Figure 12 and then performing SG filtering on each component, the decomposition result of improved ELMD is obtained, and the 

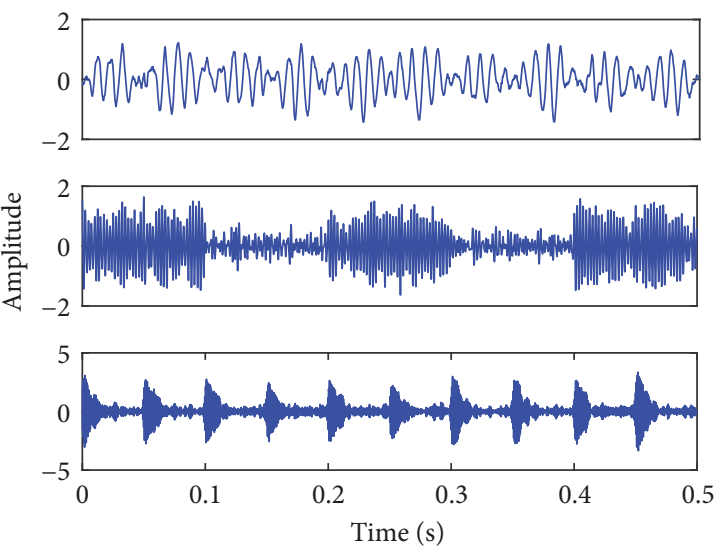

FIGURE 7: Decomposition results obtained by CELMDAN.
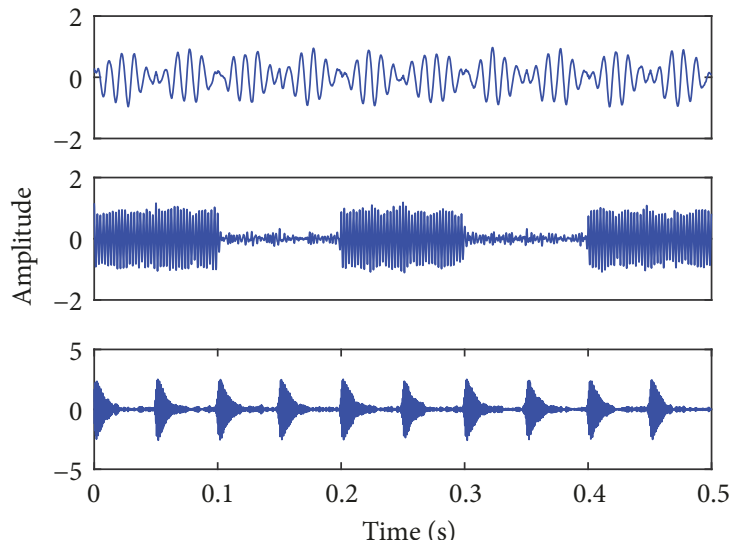

Figure 8: Decomposition results obtained by improved ELMD.

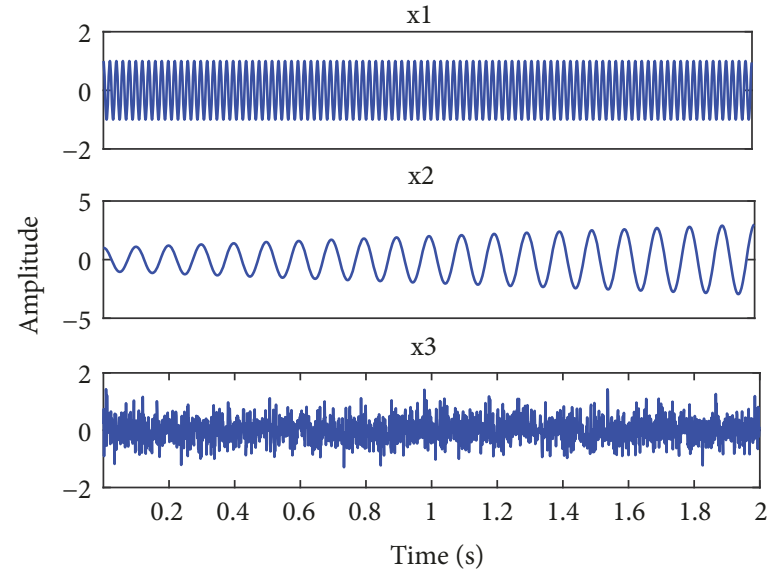

FIgURE 9: Time-domain waveform diagram of the simulated signal.

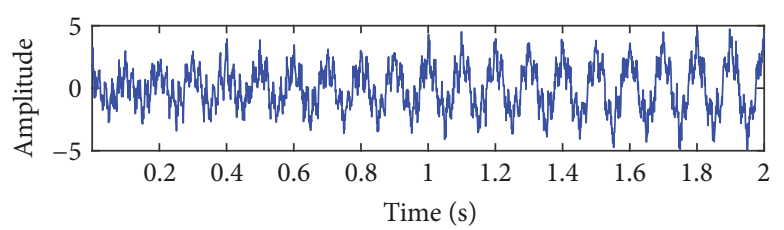

Figure 10: Time-domain waveform of the composite signal $\mathrm{x}$. 


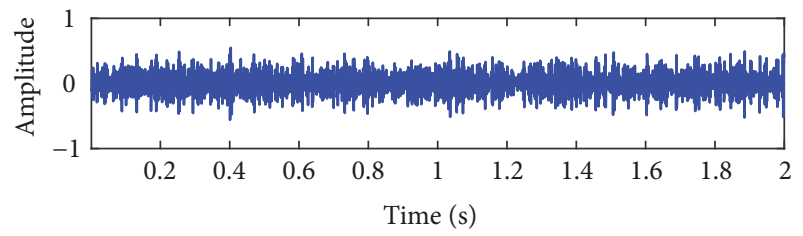

(a) P1

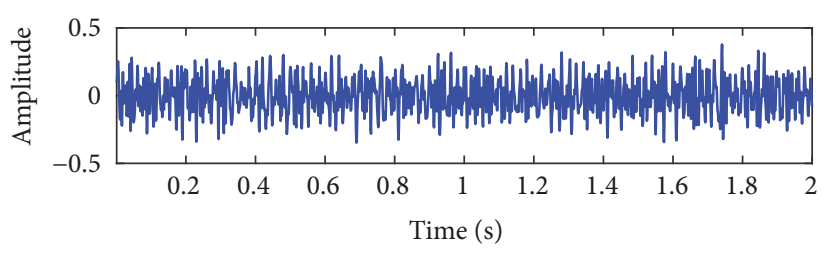

(b) P2

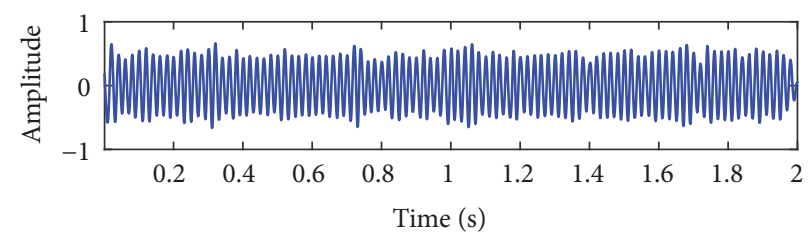

(c) P3

Figure 11: $P_{1}(t), P_{2}(t), P_{3}(t)$.

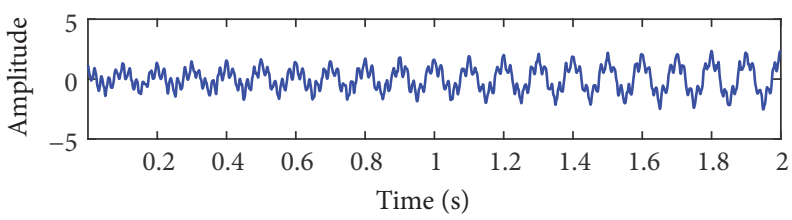

FIGURE 12: Time-domain waveform of the signal after removing the abnormal signal.

decomposition results of PF1, PF2, and residual component are shown in Figure 13(d).

The original signal is decomposed by LMD and result is shown in Figure 13(a). The first and second layers are noise, the same mode appears in the fourth and the fifth layer, and there is significant mode mixing. The sixth, seventh, and eighth layers are pseudocomponents. The original signal is decomposed by ELMD and result is shown in Figure 13(b). Figure 13(c) shows the decomposition result of CELMD. ELMD and CELMD still have pseudocomponents and the calculation amount is large. Figure 13(d) shows the decomposition result of improved ELMD. The results of the improved ELMD decomposition are PF1 and PF2, which overcomes the mode mixing phenomenon and the occurrence of pseudocomponents is avoided.

In order to further justify the superiority of the proposed method, the proposed method is compared with fault diagnosis methods based on Ensemble Empirical Mode Decomposition (EEMD) and variational mode decomposition (VMD). In gearbox fault diagnosis, EEMD and VMD are commonly used methods. Figure 14 shows the results of EEMD. The first and second layers are high-frequency noise, the third and fourth layers are components of $x_{1}$, and the fifth and sixth layers are components of $x_{2}$. Compared with the proposed method, the results of EEMD are not only more mode mixing but also more pseudocomponents. This is easy to understand that the proposed method is better than EEMD. Figure 15 shows the results of VMD. Because the simulation signal has only two meaningful components, the decomposition level in VMD is set to 2. As shown in Figure 15, the first layer contains component $\mathrm{x} 1$ and component $\mathrm{x} 2$ (compared with Figure 12), and the second layer contains a large amount of noise. It is obvious that the proposed method is better than the VMD method.

\section{Experimental Results}

4.1. Experiment 1. In order to show the effectiveness and feasibility of the proposed method in engineering practice, the relevant experiments on closed power flow gearbox test bench are carried out in this paper. In the experiment, the gearbox was loaded by the internal force generated by the torsion bar. The speed of gearbox is adjusted by controlling the electromagnetic speed regulating asynchronous motor, and the regulation range is $120 \mathrm{r} / \mathrm{min}-1200 \mathrm{r} / \mathrm{min}$. The gear transmission test bench is shown in Figure 16. The experimental devices of the test bench mainly include test bearings, rotational speed displays, motors, test gears, rotating shafts, and three-way acceleration sensors. The experimental bearing model is 32212, and the three-way acceleration sensor model is YD77SA (sensitivity is $0.01 \mathrm{~V} / \mathrm{ms}^{2}$ ). The faulty bearing is at the three-way acceleration sensor $1 \#$. The fault frequency of the rolling element is $72 \mathrm{~Hz}$, the fault frequency of the outer ring of/bearing is $160 \mathrm{~Hz}$, and the meshing frequency of the gear is $360 \mathrm{~Hz}$. The number of sampling points is 2048 . This paper takes the composite fault as an example to verify the feasibility of the improved ELMD method. There are three composite faults, namely, gear peeling, bearing outer ring defects, and rolling element defects, and the simulated fault of bearing is shown in Figure 17. Figure 17(a) shows the fault of bearing ball, and Figure 17(a) shows the fault of outer 


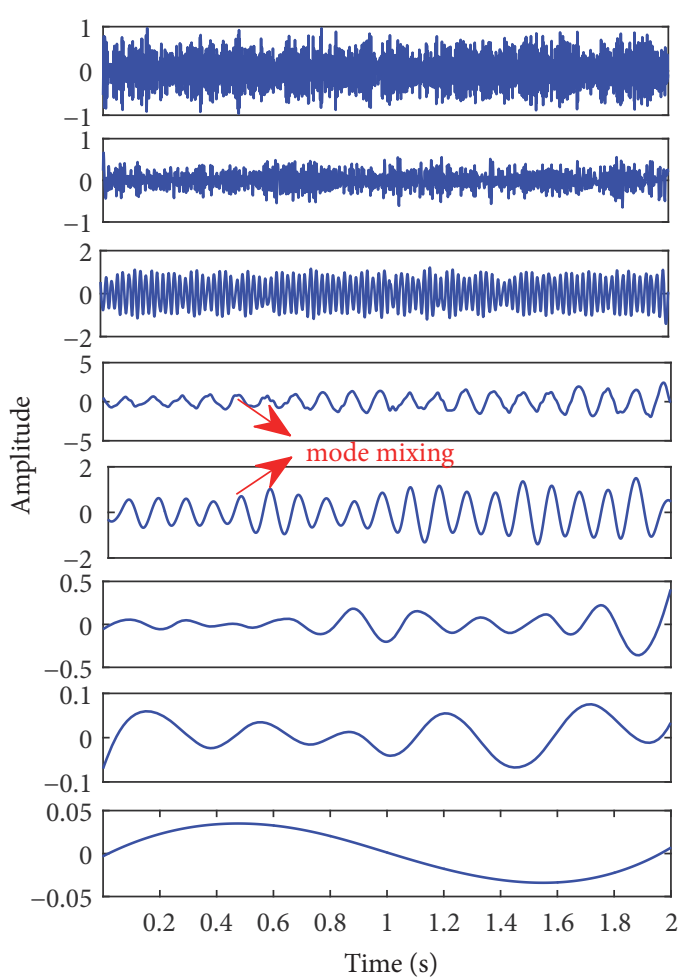

(a)

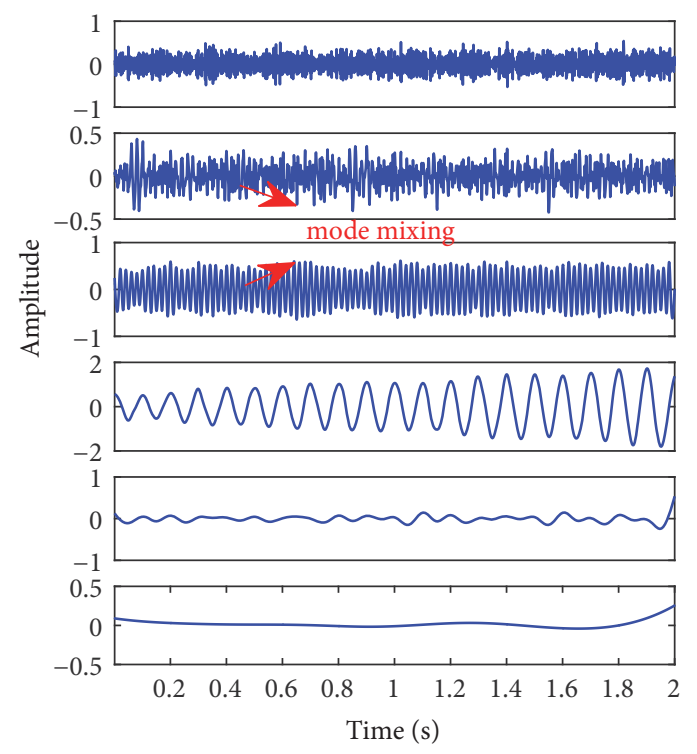

(c)

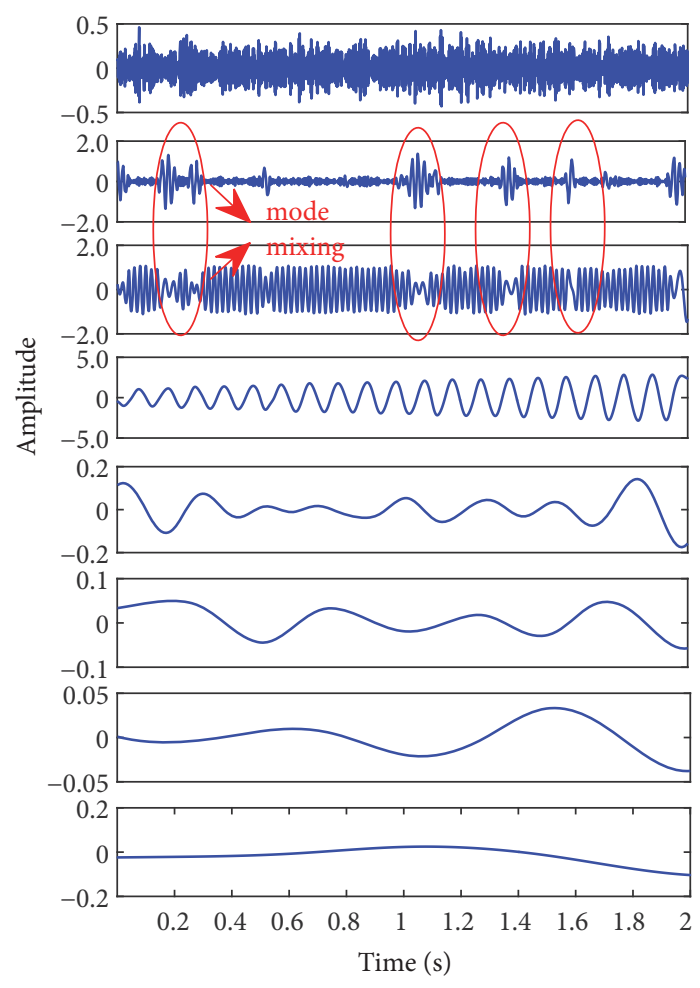

(b)

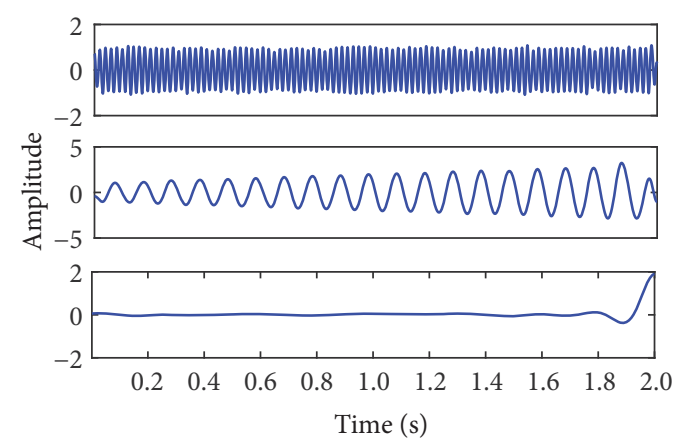

(d)

FIGURE 13: Decomposition results obtained by LMD (a), ELMD (b), CELMD (c), and improved ELMD (d).

ring. Some data are shown in Table 5. Bearing parameters are shown in Table 6.

The time-domain waveform of the vibration signal and its spectrum diagram are shown in Figure 18. Figure 18(a) shows the time-domain waveform and Figure 18(b) shows the frequency-domain waveform. There are obvious peaks in 360 $\mathrm{Hz}, 720 \mathrm{~Hz}, 160 \mathrm{~Hz}$, which are meshing frequency and it is multiple of gear and bearing outer ring frequency. However, the rolling element vibration information does not protrude in the spectrum due to the presence of noise, so it is necessary to adaptively decompose the original vibration signal. The signal is decomposed by CELMD, and the obtained result is shown in Figure 19. Figure 19(a) shows the time-domain waveform and Figure 19(b) shows the frequency-domain waveform. The original signal is adaptively decomposed into 8 layers. In addition to the high-frequency noise, the 


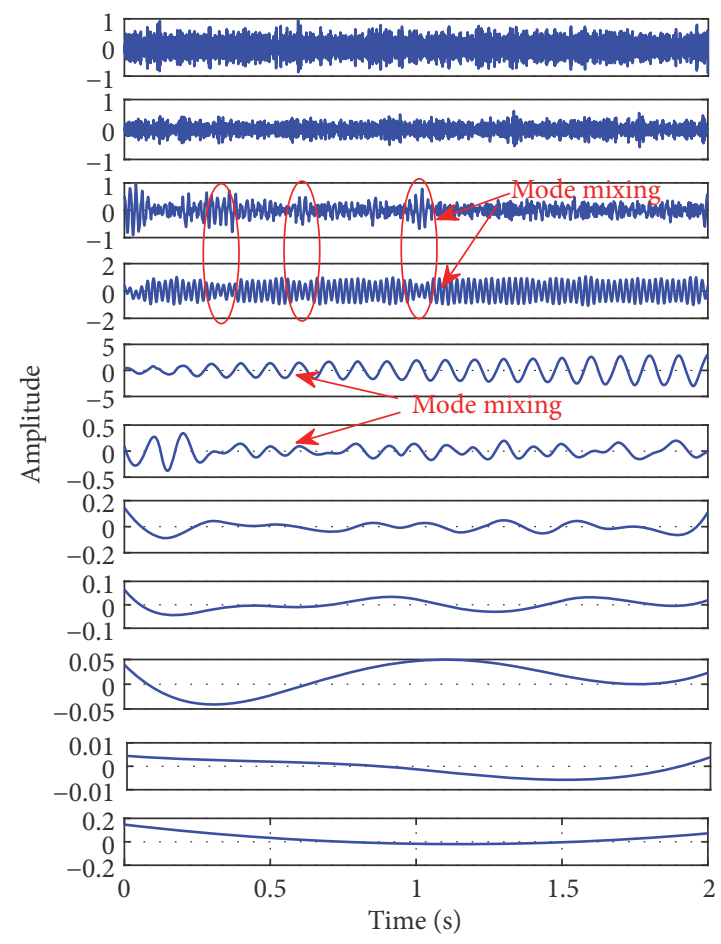

FIGURE 14: The result obtained by EEMD.

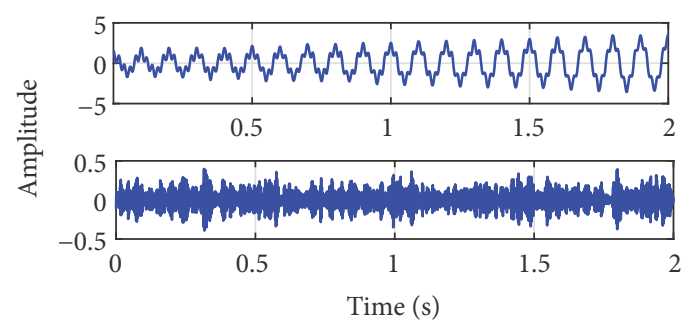

FIGURE 15: The result obtained by VMD.

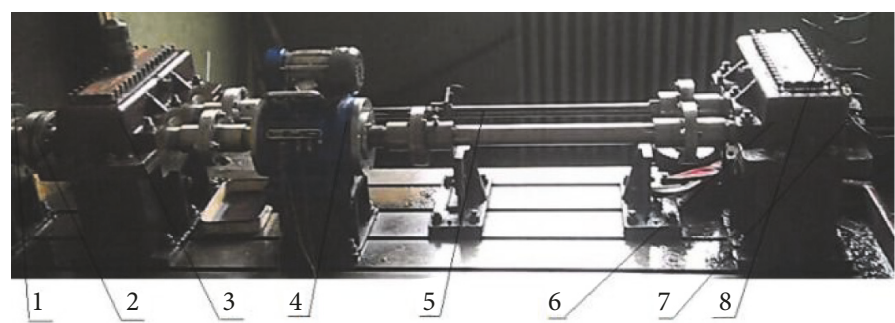

1-Speed motor, 2-Coupling, 3-Common test gearbox, 4Rotational torsion, 5-Torque bar, 6-Test gearbox, 7-Threeway acceleration sensor 1\#, 8-Three-way acceleration Sensor 2\#

Figure 16: Gear Drive Test Bench.

TABLE 5: Gear transmission test bench parameters.

\begin{tabular}{lccc}
\hline Meshing method & Transmission ratio & Sampling frequency & Sampling Point Number \\
\hline Half tooth engagement & $1: 1$ & $8 \mathrm{KHz}$ & 2000 \\
\hline Gear tooth number & Speed & Axis rotation & Load torque \\
\hline 18 & $1200 \mathrm{rpm}$ & $20 \mathrm{~Hz}$ & $1000 \mathrm{~N} \cdot \mathrm{m}$ \\
\hline
\end{tabular}




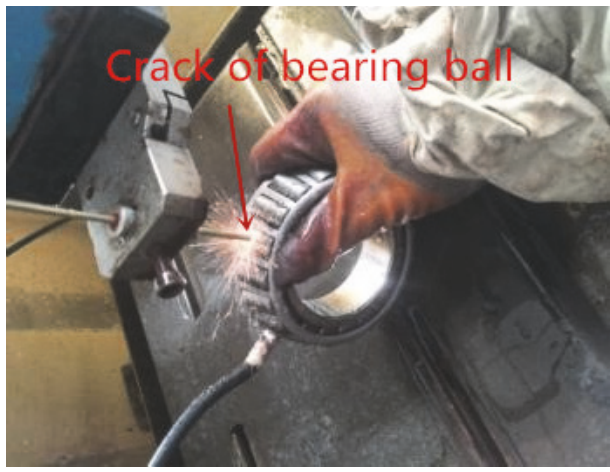

(a) Fault of bearing ball

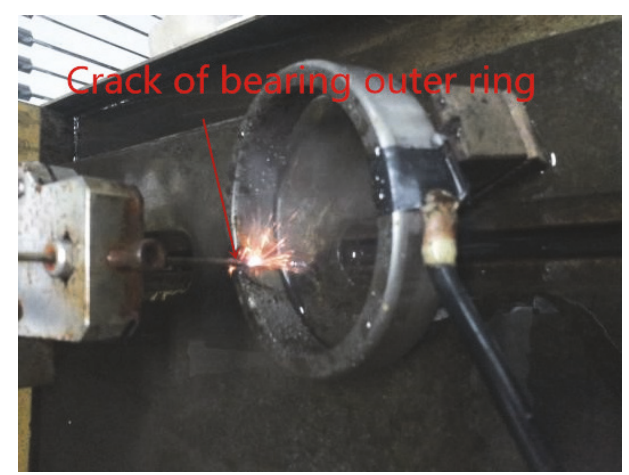

(b) Fault of outer ring

FIGURE 17: Fault bearing.

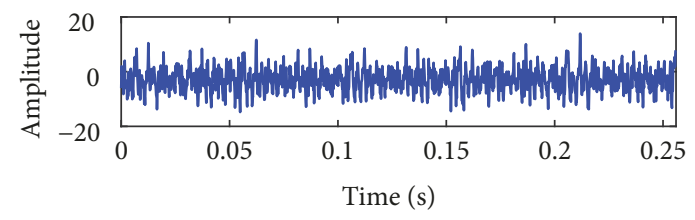

(a) Time-domain waveform

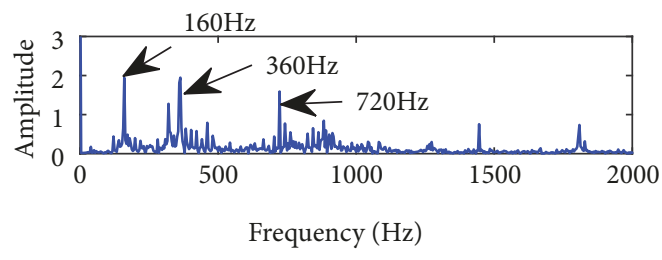

(b) Frequency-domain waveform

FIGURE 18: Time domain and spectrum analysis results of measured signals.

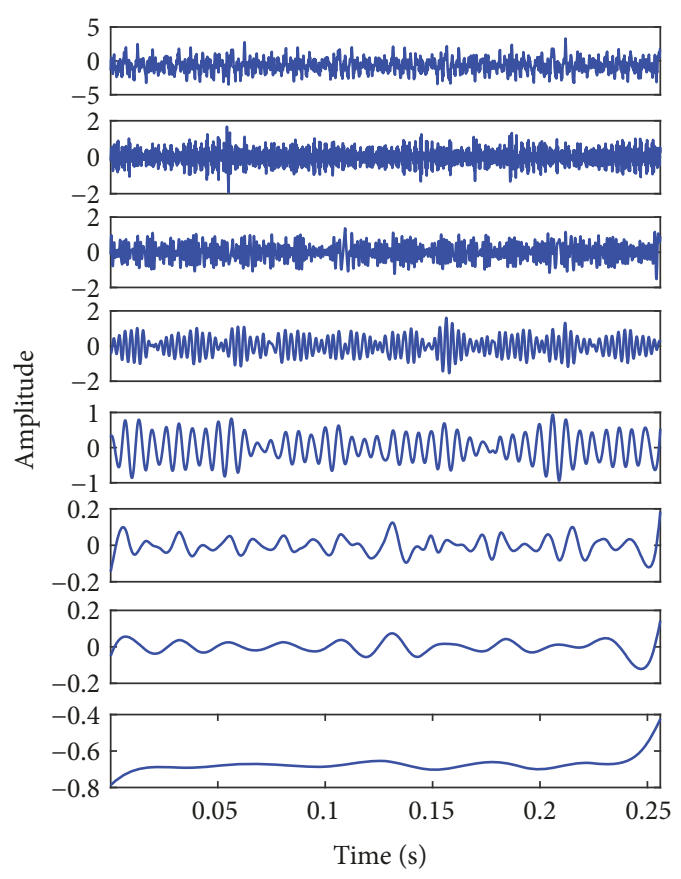

(a) Time-domain waveform

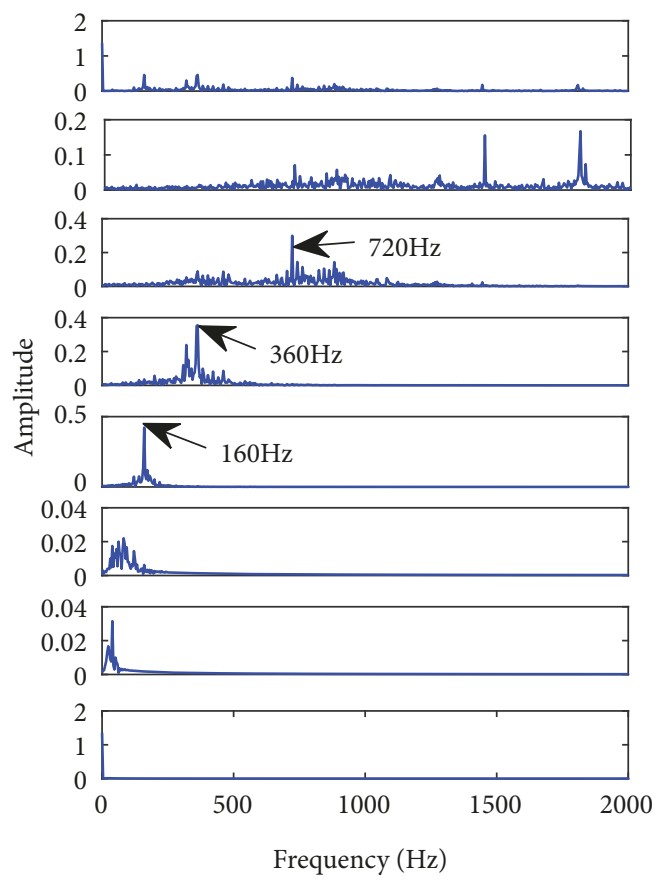

(b) Frequency-domain waveform

FIGURE 19: Decomposition results of measured signals obtained by CELMD.

TABLE 6: Bearing parameters.

\begin{tabular}{lccc}
\hline Model & Inner diameter $/ \mathrm{mm}$ & Outside diameter $/ \mathrm{mm}$ & Thickness $/ \mathrm{mm}$ \\
\hline 32212 & 60 & 110 & 28 \\
\hline
\end{tabular}




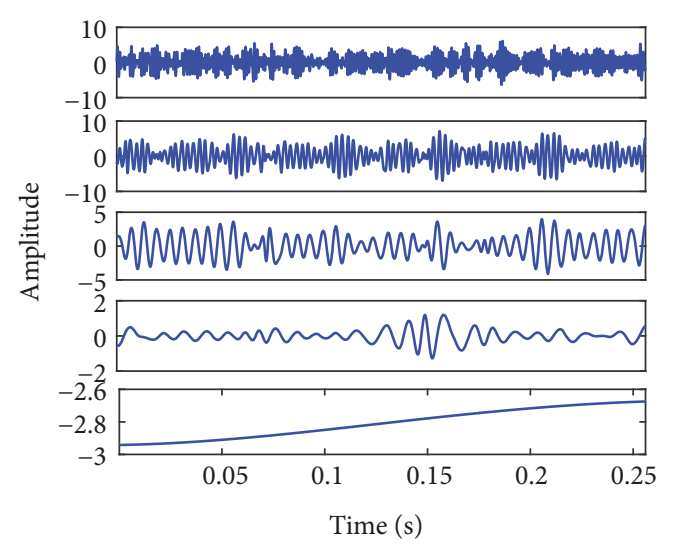

(a) Time-domain waveform

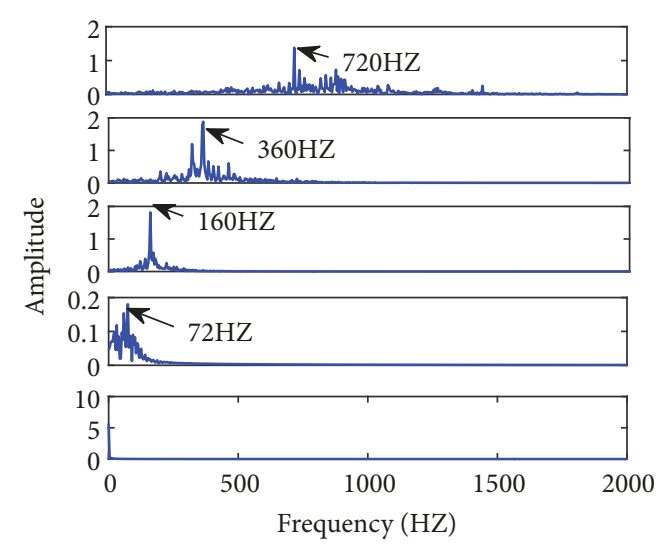

(b) Frequency-domain waveform

FIGURE 20: Decomposition results obtained by improved ELMD.

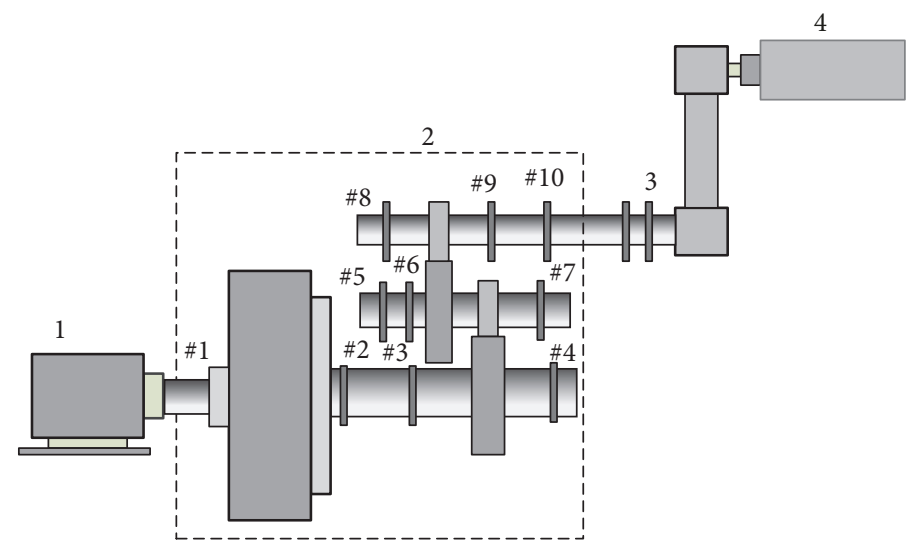

FIGURE 21: Schematic of wind turbine gearbox test bench.

first three layers exhibit mode mixing, of which $720 \mathrm{~Hz}$ is in the first three layers, and the low signal-to-noise ratio of the first two layers leads to weaker fault information energy, which is prone to misdiagnosis. The fourth and fifth layers of meshing frequency and outer ring characteristic frequency are extracted, but the sixth and seventh layers are not recognized. A specific physical meaning, the $72 \mathrm{~Hz}$ weak fault is still not extracted. Further analysis of the original signal by improved ELMD is shown in Figure 20. Figure 20(a) shows the time-domain waveform and Figure 20(b) shows the frequency-domain waveform. This method decomposes the original vibration signal into 5 layers, of which $720 \mathrm{~Hz}, 360$ $\mathrm{Hz}, 160 \mathrm{~Hz}$, and $72 \mathrm{~Hz}$, respectively, exist in different PFs. It shows that the method not only overcomes the mode mixing phenomenon, but also separates the three fault characteristics of the original signal, which further indicates that the method largely suppresses the mode mixing phenomenon and does not have pseudocomponents.

4.2. Experiment 2. In order to further verify the feasibility of the proposed method in engineering applications, experiments using a wind turbine gearbox test bench were carried out in this paper. The detailed introduction of the experimental bench is shown in [29]. Schematic of the wind turbine gearbox test bench is shown in Figure 21. In the test, bearing with inner ring fault on shaft \#10 and bearing with rolling element fault on shaft $\# 8$ are adopted. In addition, the high-speed shaft has slight bending. Information of fault frequency is shown in Table 7. Figure 22 shows the vibration signal. Figure 22(a) shows the time-domain waveform and Figure 22(b) shows the frequency-domain waveform. From the frequency-domain diagram, it can be found that the fault information is not obvious and there is no fault frequency about slight bending of high-speed shaft $(28 \mathrm{~Hz})$. This is because the collected data contains a lot of noise.

The signal is decomposed by CELMD, and the obtained result is shown in Figure 23. Figure 23(a) shows the timedomain waveform and Figure 23(b) shows the frequencydomain waveform. The original signal is adaptively decomposed into 7 layers. Among them, the first three levels are the components of high-frequency noise. The fourth layer is fault frequency about rolling element fault on shaft \#8. The first layer is fault frequency about inner ring fault on shaft \#10. The latter two levels are meaningless components. However, the fault frequency about slight bending of highspeed shaft was not extracted. Further analysis of the original 


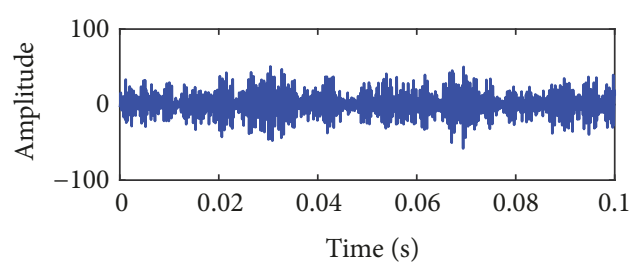

(a) Time-domain waveform

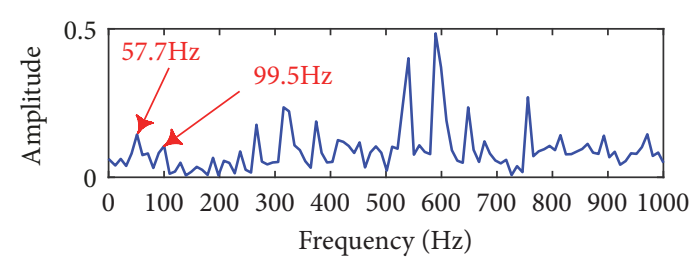

(b) Frequency-domain waveform

FIGURE 22: Time domain and spectrum analysis results of measured signals.

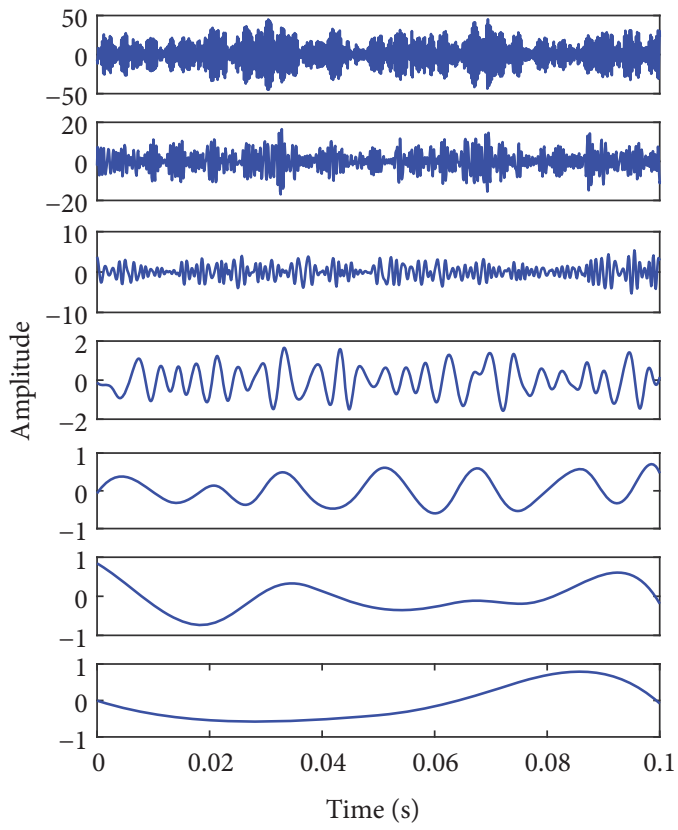

(a) Time-domain waveform

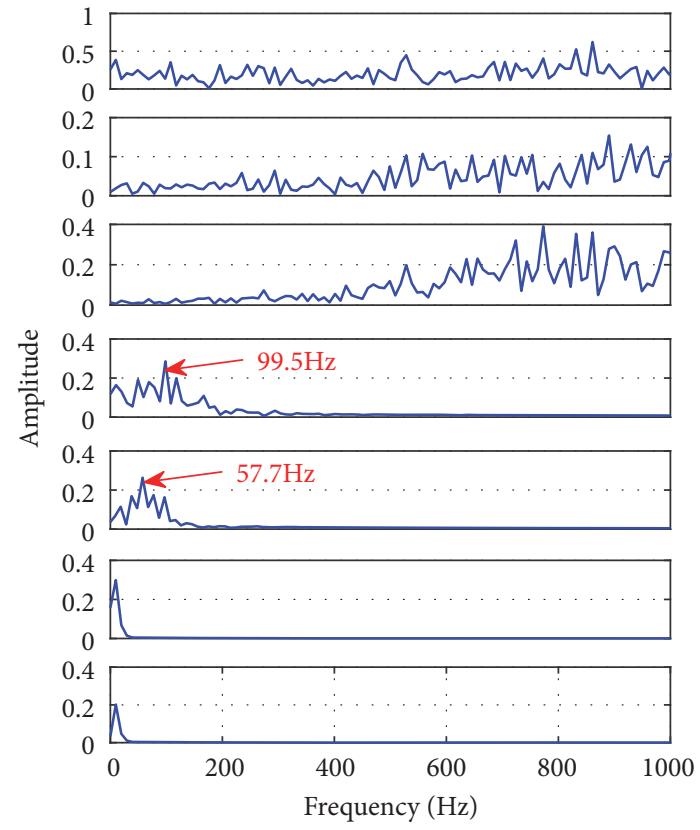

(b) Frequency-domain waveform

FiguRE 23: CELMD decomposition results of measured signals.

signal by improved ELMD is shown in Figure 24. Figure 24(a) shows the time-domain waveform and Figure 24(b) shows the frequency-domain waveform. The original signal is decomposed into 4 layers. The first layer is fault frequency about rolling element fault on shaft \#8. The second layer is fault frequency about inner ring fault on shaft $\# 10$. The third layer is fault frequency about slight bending of high-speed shaft. It can be seen that all the fault information is extracted through the method proposed in this paper, which verifies the effectiveness of the proposed method in the application.

\section{Discussion}

In this paper, the proposed method is verified by simulation and experiment. This paper verifies the proposed method through simulation and experiment. In the simulation, three signals were constructed and processed with LMD, ELMD, CELMD, and improved ELMD, respectively. The decomposition results obtained by LMD, ELMD, and CELMD show different degrees of modal aliasing and more pseudocomponents. The improved ELMD perfectly decomposes three signals, overcoming mode mixing and pseudocomponents.

In the experiment, vibration signals including gear peeling, bearing outer ring defects, rolling element defects were collected and processed by various methods. The proposed method successfully extracts three fault characteristic frequencies and has no mode mixing and pseudocomponents. However, CELMD and ELMD were unable to extract the rolling element fault characteristic frequency of $72 \mathrm{~Hz}$. In another test, the fault included bearing inner ring failure and rolling element failure. In addition, the high-speed shaft is slightly curved. The proposed method successfully extracts three fault characteristic frequencies and has no mode mixing and pseudocomponents. However, CELMD cannot extract shaft bending faults.

Through the comparison and analysis of simulation and experiment, it can be concluded that the proposed method can restrain the mode mixing and pseudocomponent, and the noise reduction effect is better than CELMD and ELMD. 
TABLE 7: Fault information.

\begin{tabular}{lccc}
\hline Fault location & Rotational frequency of shaft. & Inner ring fault on shaft \#10 & Rolling element fault on shaft \#8 \\
\hline Fault frequency & 28 & 57.7 & 99.5 \\
\hline
\end{tabular}

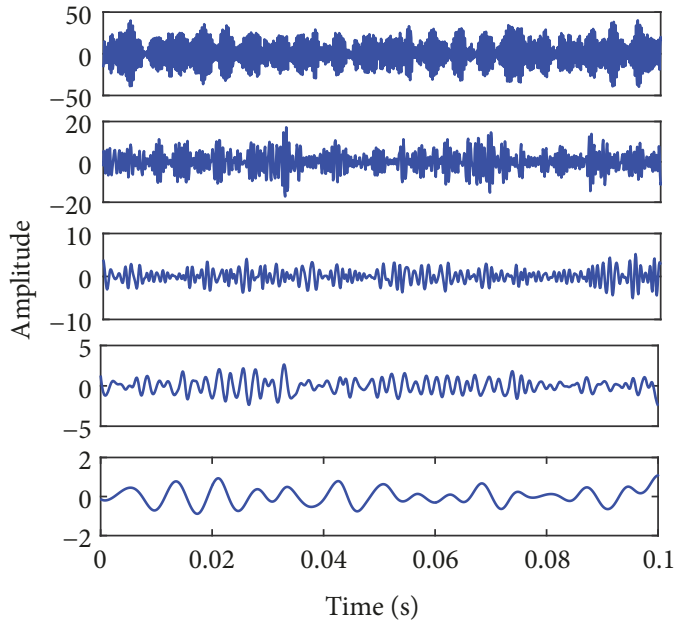

(a) Time-domain waveform

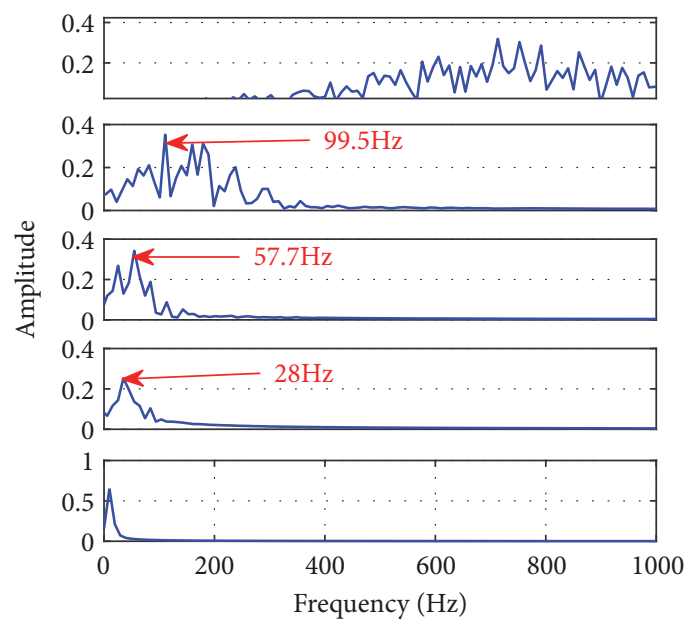

(b) Frequency-domain waveform

FIGURE 24: Improved ELMD decomposition results.

\section{Conclusion}

The objective of this paper is to make an attempt to solve the problem of difficulty faced while extracting fault features accurately under strong noise background. To address this issue, the paper studies local mean decomposition and its development, which is a new time-frequency decomposition technology. The mode mixing generated by intermittent signals is the problem of local mean decomposition (LMD). Based on the noise-assisted method, ensemble local mean decomposition (ELMD) method alleviates the mode mixing problem of LMD to some extent, but the added white noise cannot be completely neutralized. By CELMDAN, the energy of each added noise can be determined adaptively, but it ignores the effects of high-frequency noise in the original signal during the decomposition process.

This paper proposes an improved ELMD method to extract composite fault feature of gearbox. By combining CELMD (Complementary ELMD) and Permutation Entropy (PE), the high noise components can be eliminated directly. The PF component is obtained by smoothing the low noise component combined with S-G filtering. This method overcomes the mode mixing phenomenon and reduces the reconstruction error. Moreover, the occurrence of pseudocomponents is also avoided, and the amount of calculation is reduced. Characteristic information is extracted effectively in both simulation analysis and experimental analysis, and the feasibility of the method is illustrated in comparison to LMD and CELMD.

\section{Data Availability}

The data used to support the findings of this study are available from the corresponding author upon request.

\section{Conflicts of Interest}

The authors declare that they have no conflicts of interest.

\section{Acknowledgments}

This work was supported by Shanxi Applied Basic Research Project (Grant No. 201801D221237), the project supported by Science Foundation of North University of China (No. XJJ201802), and the Shanxi Applied Basic Research Project (Grant No. 201801D121186).

\section{References}

[1] B. Zhao, S. Zhang, J. Man, Q. Zhang, and Y. Chen, "A modified normal contact stiffness model considering effect of surface topography," Proceedings of the Institution of Mechanical Engineers, Part J: Journal of Engineering Tribology, vol. 229, no. 6, pp. 677-688, 2015.

[2] X. Guo, J. Tang, J. Li, C. Wang, C. Shen, and J. Liu, "Determine turntable coordinate system considering its non-orthogonality," Review of Scientific Instruments, vol. 90, no. 3, Article ID 033704, 2019. 
[3] C. Shen, J. Yang, J. Tang, J. Liu, and H. Cao, "Note: Parallel processing algorithm of temperature and noise error for microelectro-mechanical system gyroscope based on variational mode decomposition and augmented nonlinear differentiator," Review of Scientific Instruments, vol. 89, no. 7, Article ID 076107, 2018.

[4] Y. Hao, L. Song, L. Cui, and H. Wang, "A three-dimensional geometric features-based SCA algorithm for compound faults diagnosis," Measurement, vol. 134, pp. 480-491, 2019.

[5] H. Cao, Y. Zhang, Z. Han et al., "Pole-zero-temperature compensation circuit design and experiment for dual-mass mems gyroscope bandwidth expansion," IEEE/ASME Transactions on Mechatronics, 2019.

[6] H. Cao, Y. Zhang, C. Shen et al., "Temperature energy influence compensation for mems vibration gyroscope based on RBF NN-GA-KF method," Shock and Vibration, vol. 2018, Article ID 2830686, 10 pages, 2018.

[7] B. Zhao, S. Zhang, Q. F. Wang, Q. Zhang, and P. Wang, "Loading and unloading of a power-law hardening spherical contact under stick contact condition," International Journal of Mechanical Sciences, vol. 94-95, pp. 20-26, 2015.

[8] H. Y. Liu, W. G. Huang, S. B. Wang, and Z. K. Zhu, "Adaptive spectral kurtosis filtering based on Morlet wavelet and its application for signal transients detection," Signal Processing, vol. 96, pp. 118-124, 2014.

[9] S. Zhang, Z. Sun, M. Wang, J. Long, Y. Bai, and C. Li, “Deep fuzzy echo state networks for machinery fault diagnosis," IEEE Transactions on Fuzzy Systems, pp. 1-1, 2019.

[10] X. Jiang, J. Wang, J. Shi, C. Shen, W. Huang, and Z. Zhu, "A coarse-to-fine decomposing strategy of VMD for extraction of weak repetitive transients in fault diagnosis of rotating machines," Mechanical Systems and Signal Processing, vol. 116, pp. 668-692, 2019.

[11] J. Cai and Y. Xiao, "Time-frequency analysis method of bearing fault diagnosis based on the generalized S transformation," Journal of Vibroengineering, vol. 19, no. 6, pp. 4221-4230, 2017.

[12] N. Li, W. Huang, W. Guo, G. Gao, and Z. Zhu, "Multiple enhanced sparse decomposition for gearbox compound fault diagnosis," IEEE Transactions on Instrumentation and Measurement, pp. 1-12, 2019.

[13] X. Jiang, S. Li, and C. Cheng, "A novel method for adaptive multiresonance bands detection based on VMD and using MTEO to enhance rolling element bearing fault diagnosis," Shock and Vibration, vol. 2016, Article ID 8361289, 20 pages, 2016.

[14] Z. Wang, W. Du, J. Wang et al., "Research and application of improved adaptive MOMEDA fault diagnosis method," Measurement, vol. 140, pp. 63-75, 2019.

[15] H. Wang, P. Wang, L. Song, B. Ren, and L. Cui, "A novel feature enhancement method based on improved constraint model of online dictionary learning," IEEE Access, vol. 7, pp. 17599-17607, 2019.

[16] C. Shen, X. Liu, H. Cao et al., "Brain-like navigation scheme based on MEMS-INS and place recognition," Applied Sciences, vol. 9, no. 8, p. 1708, 2019.

[17] S. Lu, Q. He, and J. Wang, "A review of stochastic resonance in rotating machine fault detection," Mechanical Systems and Signal Processing, vol. 116, pp. 230-260, 2019.

[18] Z. Wang, J. Zhou, J. Wang et al., "A novel fault diagnosis method of gearbox based on maximum kurtosis spectral entropy deconvolution," IEEE Access, vol. 7, pp. 29520-29532, 2019.
[19] L. Song, H. Wang, and P. Chen, "Step-by-step Fuzzy diagnosis method for equipment based on symptom extraction and trivalent logic fuzzy diagnosis theory," IEEE Transactions on Fuzzy Systems, vol. 26, no. 6, pp. 3467-3478, 2018.

[20] M. A. Colominas, G. Schlotthauer, and M. E. Torres, "Improved complete ensemble EMD: a suitable tool for biomedical signal processing," Biomedical Signal Processing and Control, vol. 14, no. 1, pp. 19-29, 2014.

[21] Z. Yuqing, L. Xinfang, L. Fengping, S. Bingtao, and X. Wei, "An online damage identification approach for numerical control machine tools based on data fusion using vibration signals," Journal of Vibration and Control, vol. 21, no. 15, pp. 2925-2936, 2015.

[22] X. Jiang, C. Shen, J. Shi, and Z. Zhu, "Initial center frequencyguided VMD for fault diagnosis of rotating machines," Journal of Sound and Vibration, vol. 435, pp. 36-55, 2018.

[23] Z. Wang, G. He, W. Du et al., "Application of parameter optimized variational mode decomposition method in fault diagnosis of gearbox," IEEE Access, vol. 7, pp. 44871-44882, 2019.

[24] W. Huang, G. Gao, N. Li, X. Jiang, and Z. Zhu, "Timefrequency squeezing and generalized demodulation combined for variable speed bearing fault diagnosis," IEEE Transactions on Instrumentation and Measurement, pp. 1-11, 2018.

[25] J. Yeh, J. Shieh, and N. E. Huang, "Complementary ensemble empirical mode decomposition: a novel noise enhanced data analysis method," Advances in Adaptive Data Analysis (AADA), vol. 2, no. 2, pp. 135-156, 2010.

[26] J. S. Smith, "The local mean decomposition and its application to EEG perception data," Journal of the Royal Society Interface, vol. 2, no. 5, pp. 443-454, 2005.

[27] Y. Gao, F. Villecco, M. Li, and W. Song, "Multi-scale Permutation entropy based on improved LMD and HMM for rolling bearing diagnosis," Entropy, vol. 19, no. 4, article 176, 2017.

[28] X. Guo, J. Tang, J. Li, C. Shen, and J. Liu, "Attitude measurement based on imaging ray tracking model and orthographic projection with iteration algorithm," ISA Transactions, 2019.

[29] Z. Wang, Z. Han, F. Gu, J. X. Gu, and S. Ning, "A novel procedure for diagnosing multiple faults in rotating machinery," ISA Transactions, vol. 55, pp. 208-218, 2015.

[30] Y. Wang, Z. He, and Y. Zi, "A decomposition method based on improved local mean decomposition and its application in rubimpact fault diagnosis," Measurement Science \& Technology, vol. 20, no. 2, p. 28, 2009.

[31] H. Liu and M. Han, "A fault diagnosis method based on local mean decomposition and multi-scale entropy for roller bearings," Mechanism and Machine Theory, vol. 75, pp. 67-78, 2014.

[32] R. Song and X. Chen, "Analysis of fiber optic gyroscope vibration error based on improved local mean decomposition and kernel principal component analysis," Applied Optics, vol. 56, no. 8, pp. 2265-2272, 2017.

[33] J. Zheng, H. Pan, S. Yang, and J. Cheng, "Generalized composite multiscale permutation entropy and Laplacian score based rolling bearing fault diagnosis," Mechanical Systems and Signal Processing, vol. 99, pp. 229-243, 2018.

[34] Y. Li, G. Li, Y. Yang, X. Liang, and M. Xu, "A fault diagnosis scheme for planetary gearboxes using adaptive multi-scale morphology filter and modified hierarchical permutation entropy," Mechanical Systems and Signal Processing, vol. 105, pp. 319-337, 2018. 
[35] L. Lei, "Curve smoothing and denoising based on savitzky-golay algorithm," Computer and Information Technology, vol. 22, no. 5, pp. 30-31, 2014.

[36] D. Cuesta-Frau, P. Miró-Martínez, S. Oltra-Crespo, J. JordánNúñez, B. Vargas, and L. Vigil, "Classification of glucose records from patients at diabetes risk using a combined permutation entropy algorithm," Computer Methods and Programs in Biomedicine, vol. 165, pp. 197-204, 2018.

[37] Y. Guo, G. Li, H. Chen et al., "An enhanced PCA method with Savitzky-Golay method for VRF system sensor fault detection and diagnosis," Energy and Buildings, vol. 142, pp. 167-178, 2017.

[38] C. Bandt and B. Pompe, "Permutation entropy: a natural complexity measure for time series," Physical Review Letters, vol. 88, no. 17, Article ID 174102, 2002.

[39] Y. Yang, M. Zhou, Y. Niu et al., "Epileptic seizure prediction based on permutation entropy," Frontiers in Computational Neuroscience, vol. 12, 2018.

[40] Z. Wang, J. Wang, and W. Du, "Research on fault diagnosis of gearbox with improved variational mode decomposition," Sensors, vol. 18, no. 10, p. 3510, 2018. 


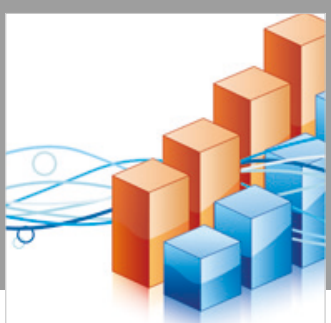

Advances in

Operations Research

\section{-n-m}
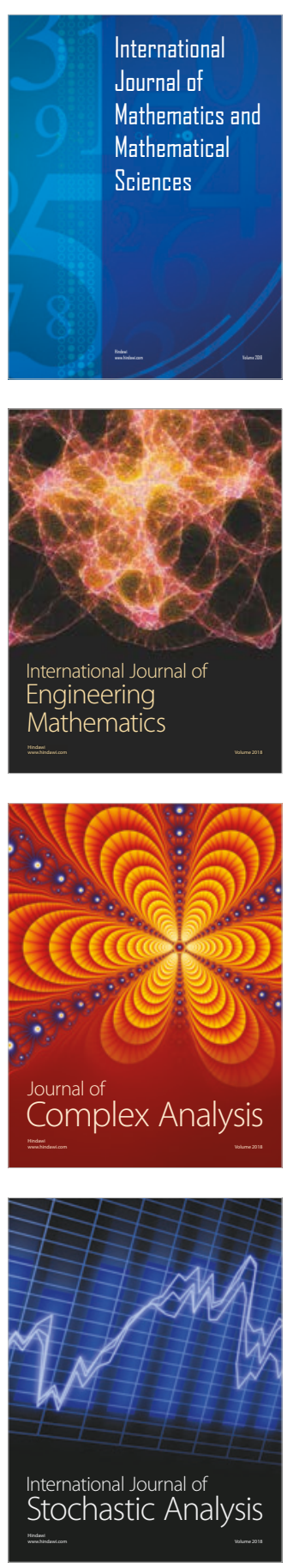
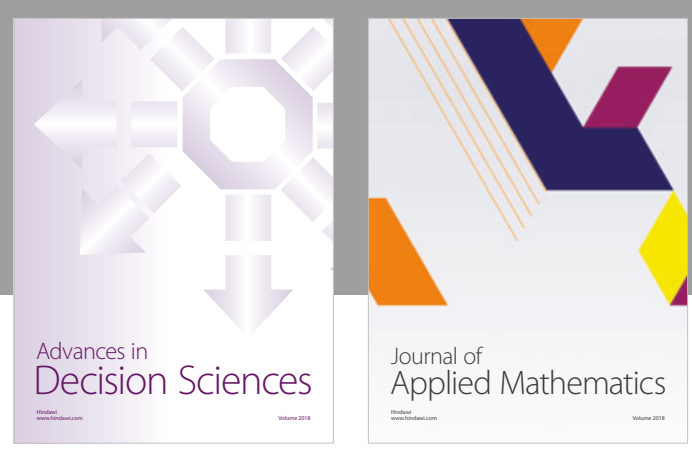

Journal of

Applied Mathematics
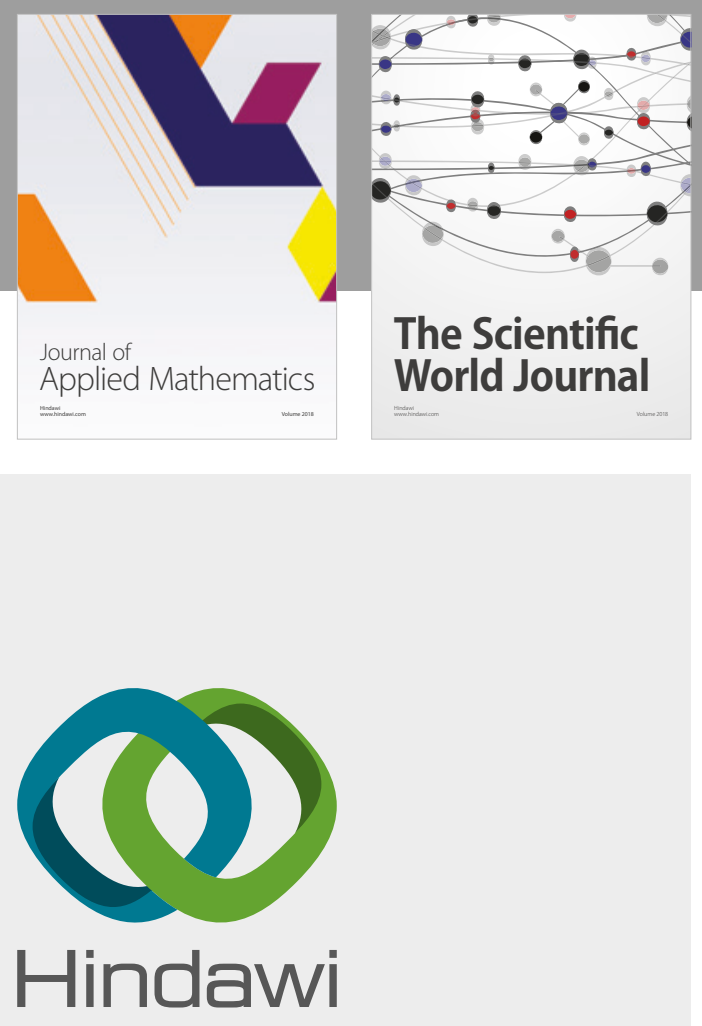

Submit your manuscripts at

www.hindawi.com

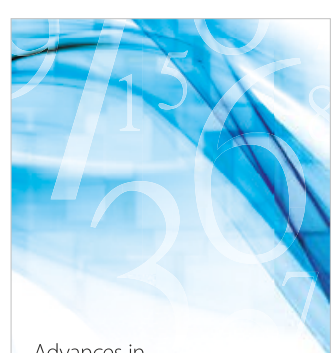

Advances in
Numerical Analysis
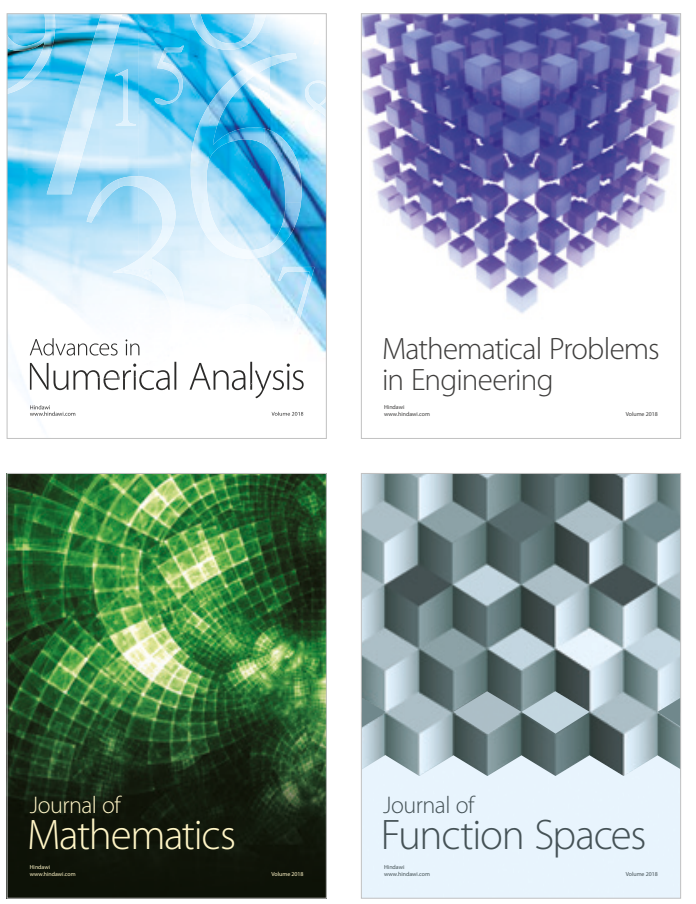

Mathematical Problems in Engineering

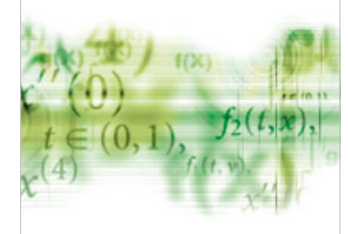

International Journal of

Differential Equations

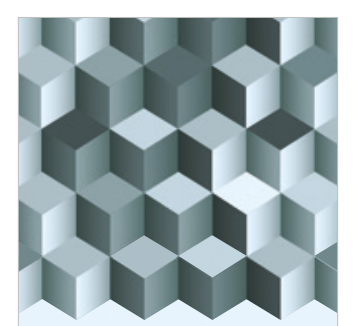

Journal of

Function Spaces
The Scientific

World Journal

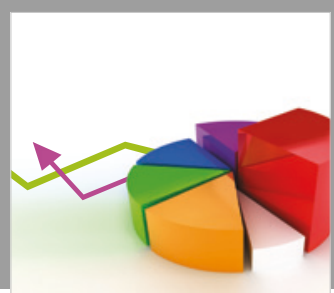

Journal of

Probability and Statistics
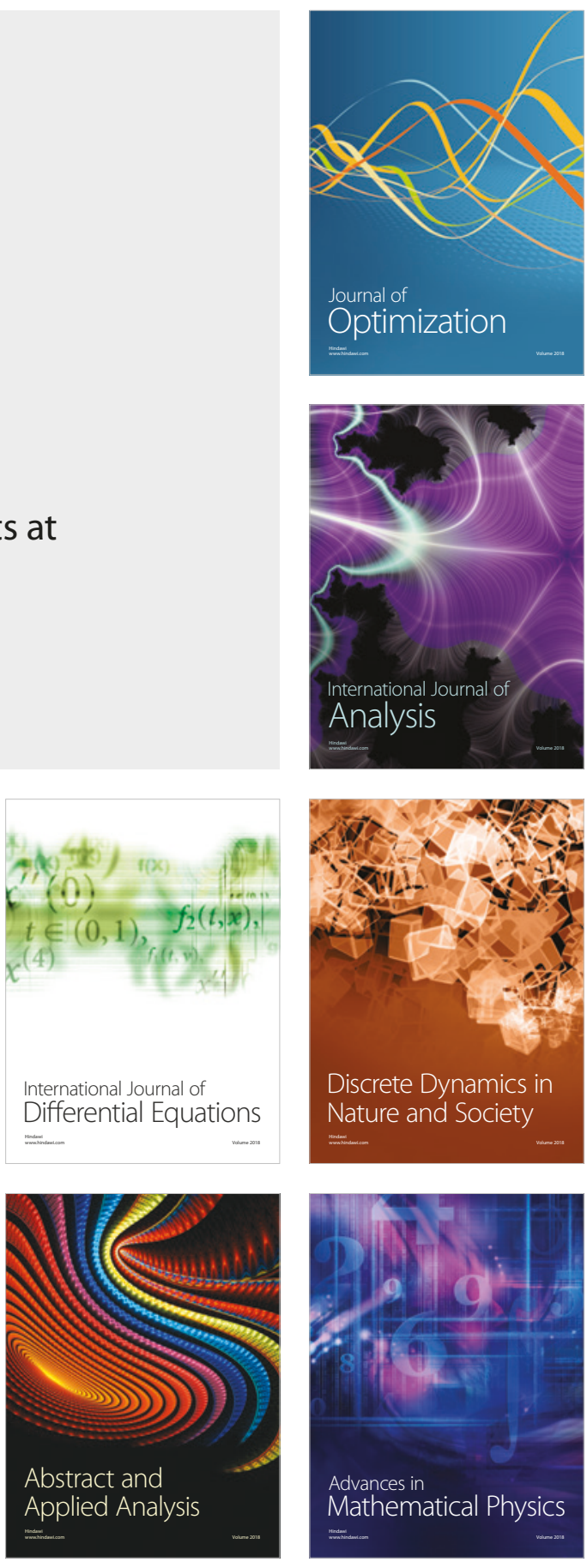